\title{
Estudio de aceptabilidad y saciedad de barritas de cereal altas en fibra dietética en escolares de una escuela rural de Chile
}

\author{
Acceptability and saciety study of cereal \\ bars high in dietetic fiber in schoolage \\ children of a Chilean rural school
}

\begin{abstract}
Cereal bars for infant collation in two flavors were developed: apple and red fruits. The bars were made with oatmeal and dried fruit, low in saturated fat, total sugars and sodium. The effect of the addition of $2.5 \%$ of $\beta$-glucan in the bars on the sensory acceptability, satiety and digestive tolerance in primary school children were evaluated. The results showed high sensory acceptability to all bars (both flavors, with and without $\beta$-glucan), being higher in children from 1 st to 4 th grade and in men $(p<0.05)$. Consumption of the bars produced satiety in children without stumbling significant differences due to the presence of $\beta$-glucan. Regarding the digestive tolerance, no cases of discomfort were presented, indicating that all cereal bars were well tolerated. The addition of $\beta$-glucan in the bars does not affect the sensory acceptability of these and does not affect satiety compared to bars without $\beta$-glucan.

Key words: $\beta$-glucan, sensory, aceptability, satiety, cereal bar.
\end{abstract}

\section{INTRODUCIÓN}

La obesidad constituye el mayor factor relacionado con diversas enfermedades crónicas que incluyen síndrome metabólico, resistencia a la insulina, diabetes tipo 2, hipertensión arterial, dislipidemia, aterosclerosis y distintas formas de cáncer (1-3). El progresivo aumento de personas obesas constituye un problema social y sanitario a nivel mundial, ya que ellas tienen mayor susceptibilidad a contraer estas enfermedades, lo que afecta seriamente su calidad de vida.

La obesidad infantil presenta una prevalencia alta y creciente tanto en países desarrollados como en vías de desarrollo. En las últimas décadas, la prevalencia de obesidad se ha duplicado o triplicado en muchos países, alcanzado en Chile cifras cercanas al $10 \%$ los primeros años de vida y superiores al $20 \%$ en escolares de $1^{\circ}$ básico, a lo que se agrega una proporción similar de niños con sobrepeso. (4).

Las conductas alimentarias de la población chilena en los diferentes grupos etarios se caracterizan por un alto consumo de alimentos procesados altos en azúcar, grasa, sodio y calorías
Vilma Quitral R. (1)

Eduardo Atalah S. (1)

María Fernanda Jara C.(1)

Francisca Echeverría G.(1)

Juan P. Vivanco L.(2)

Ximena López A.(2)

(1) Departamento de Nutrición. Facultad de Medicina. Universidad de Chile, Santiago, Chile. (2) Consorcio de Cereales Funcionales S.A. Centro de Innovación Agroalimentario de Chile, Santiago, Chile.

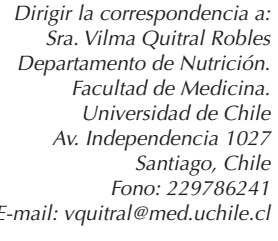

Este trabajo fue recibido el 7 de Abril de 2015 aceptado con indicaciones el 5 de Octubre de 2015 y aceptado para ser publicado el 14 de Enero de 2016. y un bajo consumo de alimentos saludables, como verduras y frutas. Los malos hábitos alimentarios se desarrollan desde los primeros años de vida, los que en general se mantienen después en la edad adulta (5-7).

Frente a este problema, se hace necesario modificar las conductas en los escolares, como promoción de alimentación saludable y aumento de actividad física, existiendo diversas experiencias exitosas tanto en Chile como en otros países (8).

Las colaciones que consumen los estudiantes generalmente son alimentos altos en calorías, azúcares o sal, lo que debería ser reemplazado por frutas $u$ otro alimento con bajo contenido de nutrientes críticos y alto aporte de fibra dietética. Alimentos como frutas, cereales altos en fibra y otros similares tienen efectos beneficiosos en la salud, como ayudar a disminuir el riesgo de enfermedades cardiovasculares, diabetes tipo 2 y otras patologías y un mayor consumo de fibra dietética provoca mayor saciedad (8-10).

La fibra dietética está formada por diferentes componentes, entre los cuales se encuentra el $\beta$-glucano, que es 
un polisacárido compuesto por moléculas de glucosa unidas por enlaces $\beta-(1 \rightarrow 4)$ y $\beta-(1 \rightarrow 3)$. Su estructura química es responsable de sus propiedades físicas, como su viscosidad y solubilidad, así como su acción sobre el metabolismo de colesterol (11). Además el aumento de la viscosidad que produce el $\beta$-glucano dentro del tracto digestivo explica su positivo efecto en la saciedad (12).

Es importante por lo tanto desarrollar y evaluar el consumo, aceptabilidad y sensación de saciedad de colaciones saludables con alto contenido de $\beta$-glucano, que podrían contribuir de alguna manera a la reducción del riesgo de obesidad en la población infantil

\section{OBJETIVOS}

Evaluar el efecto de la incorporación de $\beta$-glucanos en barritas de cereal (en 2 sabores) sobre la aceptabilidad sensorial, saciedad y tolerancia digestiva en escolares de educación básica.

\section{METODOLOGÍA}

Se desarrollaron barritas de cereal destinadas a la colación infantil en base de avena y frutos deshidratados, con bajo contenido de sodio y buena fuente de fibra dietética, en dos formatos: barritas control y barritas con $2.5 \%$ de $\beta$-glucano, cada formato a su vez se elaboró en dos versiones: manzana y frutos rojos (información nutricional se presenta en las tablas 1 y 2). El peso molecular de $\beta$-glucano que se incorporó a las barras de cereal se distribuye entre los 1.500 y 3.500 kDa, lo que equivale a un grado de polimerización entre 8.333 a 19.444. La masa de la porción de las barritas es de $30 \mathrm{~g}$ y las dimensiones son $10 \mathrm{~cm}$ de largo $\times 3 \mathrm{~cm}$ de ancho $\times 1,5 \mathrm{~cm}$ de espesor aproximadamente.

Las barritas se entregaron como colación en horario de recreo, a niños de una Escuela Municipal de la Sexta Región, para evaluar la aceptabilidad, saciedad y tolerancia digestiva. Se invitó a participar a los 265 niños de $1^{\circ}$ a $8^{\circ}$ básico matriculados en la Escuela América de Marchigüe, provincia

TABLA 1

Información nutricional de la barra con $\beta$-glucano de manzana.

\section{ETIQUETADO BARRA BETAGLUCANOS MANZANA}

INFORMACIÓN NUTRICIONAL

Energía (kcal)
Proteínas $(\mathrm{g})$
Grasa total $(\mathrm{g})$
Hidratos de carbono disponibles (g)
Azúcares totales (g)
Alcoholes de azúcares $(\mathrm{g})$
Fibra dietética total (g)
Fibra dietética soluble (g)
Betaglucanos (g)
Fibra dietética insoluble(g)
Sodio (mg)

$100 \mathrm{~g}$

292

4,84

7,88

28,08

5,32

30,18

11,90

8,40

2,50

3,50

15
Porción (30 g)

88

1,45

2,36

8,42

1,59

9,05

3,57

2,52

0,75

1,05

4

TABLA 2

Información nutricional de barra con $\beta$-glucano de frutos rojos.

INFORMACIÓN NUTRICIONAL

ETIQUETADO BARRA BETAGLUCANOS FRUTOS ROJOS

Energía $\left(^{*}\right)(\mathrm{kcal})$
Proteínas $(\mathrm{g})$
Grasa total $(\mathrm{g})$
Hidratos de carbono disponibles (g)
Azúcares totales $(\mathrm{g})$
Azúcares de alcohol $(\mathrm{g})$
Fibra dietética total $(\mathrm{g})$
Fibra dietética soluble $(\mathrm{g})$
Betaglucanos $(\mathrm{g})$
Fibra dietética insoluble $(\mathrm{g})$
Sodio (mg)

$100 \mathrm{~g}$
304
4,91
9,25
27,86
3,60
30,18
11,90
8,40
2,50
3,50
11


del Cardenal Caro, para cubrir el rango de edades de 6 a 14 años. Se solicitó la firma del consentimiento informado por parte de los padres. El tamaño final de la muestra correspondió a 210 niños, ya que hubo 55 rechazos o entrega tardía del consentimiento informado.

Cada niño fue encuestado en 4 oportunidades, analizando la aceptabilidad y saciedad de cada una de las barritas que le fueron suministradas. Se realizó un estudio de doble ciego, en que los encuestados, encuestadores e investigadores trabajaron con los productos codificados. Una vez concluidos los análisis estadísticos del estudio se identificaron los productos.

Los escolares evaluaron una muestra control (barrita sin $\beta$-glucano) y una muestra en estudio (barrita con $\beta$-glucano) con una diferencia de 1 semana. Ambas barritas correspondían al mismo sabor. Después evaluaron barritas de otro sabor, siguiendo el mismo esquema.
Para evaluar la aceptabilidad sensorial se aplicó una escala hedónica de 5 puntos (13), la que se presenta en la figura 1 y con los niños de $1^{\circ}$ a $4^{\circ}$ básico una escala gráfica (14), (figura 2 ).

Para la evaluar el efecto del consumo de la barrita en la saciedad se aplicó una Escala Visual Análoga (EVA) (15) en 3 tiempos: $t_{0}$ : antes de consumir la barrita, $t_{1}$ : inmediatamente después de consumirla y $t_{2}: 2$ horas después. Se aplicó una Escala Visual Análoga de 5 puntos, en que 1 significa "me muero de hambre" y 5 "estoy tan satisfecho que no podría comer más", como se presenta en la figura 3.

Para la evaluación de la tolerancia digestiva se realizaron grupos focales con estudiantes de distintos cursos, a través de preguntas abiertas, opiniones sobre la aceptabilidad de las barritas y la eventual presencia de síntomas relacionados con la tolerancia digestiva (constipación, meteorismo, diarrea, entre otros.).

\section{FIGURA 1}

Ficha de respuesta de aceptabilidad sensorial. Escala hedónica de 5 puntos.

Numeración

1

2

3

4

5
Escala Hedónica

Condición

Me disgusta mucho

Me disgusta

No me gusta ni me disgusta

Me gusta

Me gusta mucho

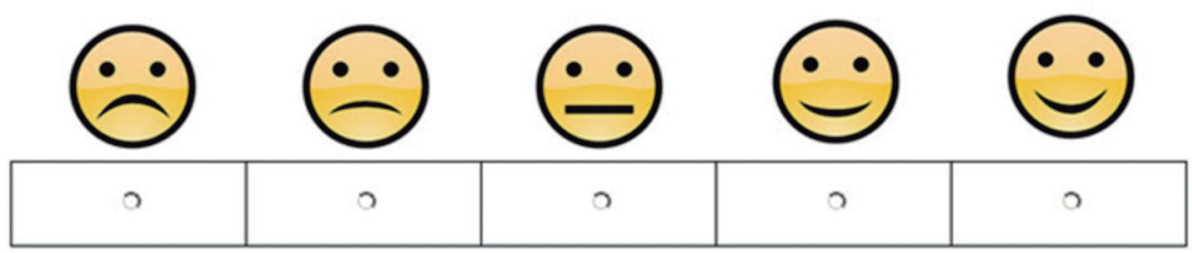

\section{FIGURA 3}

Ficha de respuesta de saciedad. Escala Visual Análoga.

\section{Numeración}

$$
1
$$

3

4

5
Escala Visual Análoga (EVA)

Condición

Me muero de hambre

Tengo un poco de hambre

No tengo hambre ni estoy satisfecho

Estoy satisfecho

Estoy tan satisfecho que no podría comer más 
Las encuestas fueron realizadas por dos nutricionistas previamente capacitadas, quienes además midieron el peso y la talla de los niños en condiciones estandarizadas y evaluó su estado nutricional según las normas de JUNAEB.

Los resultados de aceptabilidad y saciedad de cada una de las barritas se presentan como distribución de frecuencia para cada una de las 5 categorías de la escala. La aceptabilidad fue evaluada además por niveles de escolaridad ( $1^{\circ}$ a $4^{\circ}$ básico y $5^{\circ}$ a $8^{\circ}$ básico) y género.

Para analizar los cambios en la saciedad entre los tres tiempos estudiados, se calculó la diferencia en la respuesta de saciedad, informándose la mediana y el intervalo de confianza de 95\%. Los valores positivos (mayores de 0) indican un aumento de la saciedad, los valores negativos (menores de 0) indican disminución de la saciedad.

Para establecer diferencias entre los grupos se aplicó la prueba de $x 2$ de Pearson, con nivel de significación de $5 \%$. En el caso de los cambios en nivel de saciedad, se aplicó la prueba de Kruskal- Wallis. Los datos se analizaron con el programa Stata 11.1.

\section{RESULTADOS}

Del total de estudiantes del establecimiento, $40 \%$ correspondían al primer ciclo ( $1^{\circ}$ a $4^{\circ}$ básico) y $60 \%$ al segundo ciclo ( $5^{\circ}$ a $8^{\circ}$ básico), con una mayor proporción de mujeres (56\%).

La aceptabilidad sensorial es alta para todas las barritas, de acuerdo a los resultados presentados en la tabla 3. Un
$66,3 \%$ respondió "me gusta mucho", siendo mayor para las barritas de frutos rojos que de manzana. No se observó un efecto de la incorporación del $\beta$-glucano sobre la aceptabilidad de las barritas.

La tabla 4 indica que existen diferencias significativas en la aceptabilidad sensorial de las barritas de acuerdo al nivel educacional y género. La aceptabilidad fue mayor en los cursos menores ( $1^{\circ}$ a $4^{\circ}$ básico) y en varones.

De acuerdo a los datos de saciedad presentados en la tabla 5, antes de consumir la barrita $77,5 \%$ de los alumnos presentaba algún grado de hambre. La sensación de hambre era significativamente mayor en los alumnos de los cursos $1^{\circ}$ a $4^{\circ}$ ("me muero de hambre" 48,0 vs $14,6 \%$ p < 0,001 datos, no mostrados)

La sensación de hambre disminuyó en forma importante inmediatamente después del consumo de la barrita, en todos los grupos. Después de 2 horas la saciedad fue mayor que en la línea base, previo al consumo de la barrita. Un 15\% se siente satisfecho y $3,8 \%$ muy satisfecho que no podría comer más, lo que suma $18,8 \%$, mientras que antes del consumo de la barrita, este valor era 4,4\%. En el caso de las barritas de manzana es mayor la saciedad producida por el consumo de las barritas con $\beta$-glucano respecto a la barrita control, aunque las diferencias no son significativas.

En forma complementaria se analizaron los cambios promedios en el nivel de saciedad entre las tres etapas estudiadas (tabla 6): $t_{0}-t_{1}$ (pre a post consumo); $t 1-t 2$ (inmediatamente

TABLA 3

Aceptabilidad según tipo de barrita.

\begin{tabular}{|c|c|c|c|c|c|c|}
\hline \multirow{3}{*}{\multicolumn{2}{|c|}{$\begin{array}{l}\text { Sabor } \\
\text { Aceptabilidad } \\
\text { Escala hedónica de } 5 \text { puntos }\end{array}$}} & \multicolumn{2}{|c|}{ Manzana } & \multicolumn{2}{|c|}{ Frutos rojos } & \multirow[t]{3}{*}{ Total } \\
\hline & & \multirow[t]{3}{*}{ Control } & \multirow[t]{2}{*}{$\beta$-glucano } & Control & \multirow[t]{2}{*}{$\beta$-glucano } & \\
\hline & & & & & & \\
\hline & & & $\%$ & \multicolumn{2}{|c|}{$\%$} & $\%$ \\
\hline 1 & Me disgusta mucho & 2,9 & 4,7 & 2,6 & 3,1 & 2,8 \\
\hline 2 & Me disgusta & 2,9 & 2,1 & 2,6 & 4,1 & 3,4 \\
\hline 3 & No me gusta ni disgusta & 8,6 & 8,8 & 5,2 & 6,1 & 5,7 \\
\hline 4 & Me gusta & 24,1 & 28,7 & 23,6 & 20,0 & 21,8 \\
\hline \multirow[t]{2}{*}{5} & Me gusta mucho & 61,5 & 55,7 & 66,0 & 66,7 & 66,3 \\
\hline & & \multicolumn{2}{|c|}{$x 22,2 p=0,69$} & \multicolumn{2}{|c|}{$x 21,4 p=0,84$} & $x 210,9 p=0,54$ \\
\hline
\end{tabular}

TABLA 4

Aceptabilidad según nivel educacional y género.

\begin{tabular}{|c|c|c|c|c|c|}
\hline \multirow{2}{*}{\multicolumn{2}{|c|}{$\begin{array}{l}\text { Aceptabilidad } \\
\text { Escala hedónica de } 5 \text { puntos }\end{array}$}} & \multirow{3}{*}{$\begin{array}{c}1^{\circ}-4^{\circ} \text { básico } \\
\%\end{array}$} & \multirow{3}{*}{$\begin{array}{c}5^{\circ}-8^{\circ} \text { básico } \\
\%\end{array}$} & \multirow{3}{*}{$\begin{array}{c}\text { Hombre } \\
\text { \% }\end{array}$} & \multirow{3}{*}{$\begin{array}{c}\text { Mujer } \\
\%\end{array}$} \\
\hline & & & & & \\
\hline & & & & & \\
\hline 1 & Me disgusta mucho & 5,9 & 1,6 & 1,8 & 4,6 \\
\hline 2 & Me disgusta & 1,6 & 3,8 & 3,2 & 2,7 \\
\hline 3 & No me gusta ni disgusta & 2,3 & 10,5 & 6,5 & 7,7 \\
\hline 4 & Me gusta & 6,9 & 35,9 & 21,0 & 26,6 \\
\hline \multirow[t]{2}{*}{5} & Me gusta mucho & 83,3 & 48,2 & 67,5 & 58,4 \\
\hline & & \multicolumn{2}{|c|}{$x 2: 129,6 p<0,001$} & \multicolumn{2}{|c|}{$x 2: 9,8 p<0,05$} \\
\hline
\end{tabular}


después del consumo a el próximo recreo dos horas más tarde); $t_{0}-t_{2}$ (etapa previa del consumo y dos horas después). Se observó un aumento de la saciedad inmedianamente después del consumo de la barrita, tanto en la barrita control como la barrita elaborada con $\beta$-glucano. Al comparar entre 11 y t2, el valor de la mediana es el mismo, pero el intervalo de confianza indica que las barritas con $\beta$-glucano producen un aumento significativo de la saciedad.

Respecto a la tolerancia digestiva, no se presentaron casos con síntomas gastrointestinales como dolor abdominal, hinchazón, meteorismo, diarrea, entre otros. lo que indica que todas las barritas de cereal fueron bien toleradas.
DISCUSIÓN

La aceptabilidad general de las barritas fue alta, la mayoría de las respuestas correspondían a la puntuación 5 de la escala hedónica, que corresponde a "me gusta mucho". La incorporación de $\beta$-glucano no afectó la aceptabilidad, como sí ocurrió en el estudio de Volikakis y colaboradores (16) en que la incorporación de $\beta$-glucano en quesos afectó el sabor, aroma y algunas características de textura en forma significativa. Igualmente en productos cárnicos la incorporación de $\beta$-glucano afectó negativamente el sabor, color, textura y aceptabilidad general $(17,18)$.

La incorporación de $\beta$-glucano en pan, afecta el color de la

TABLA 5

Nivel de saciedad pre, post y dos horas después del consumo según tipo de barrita.

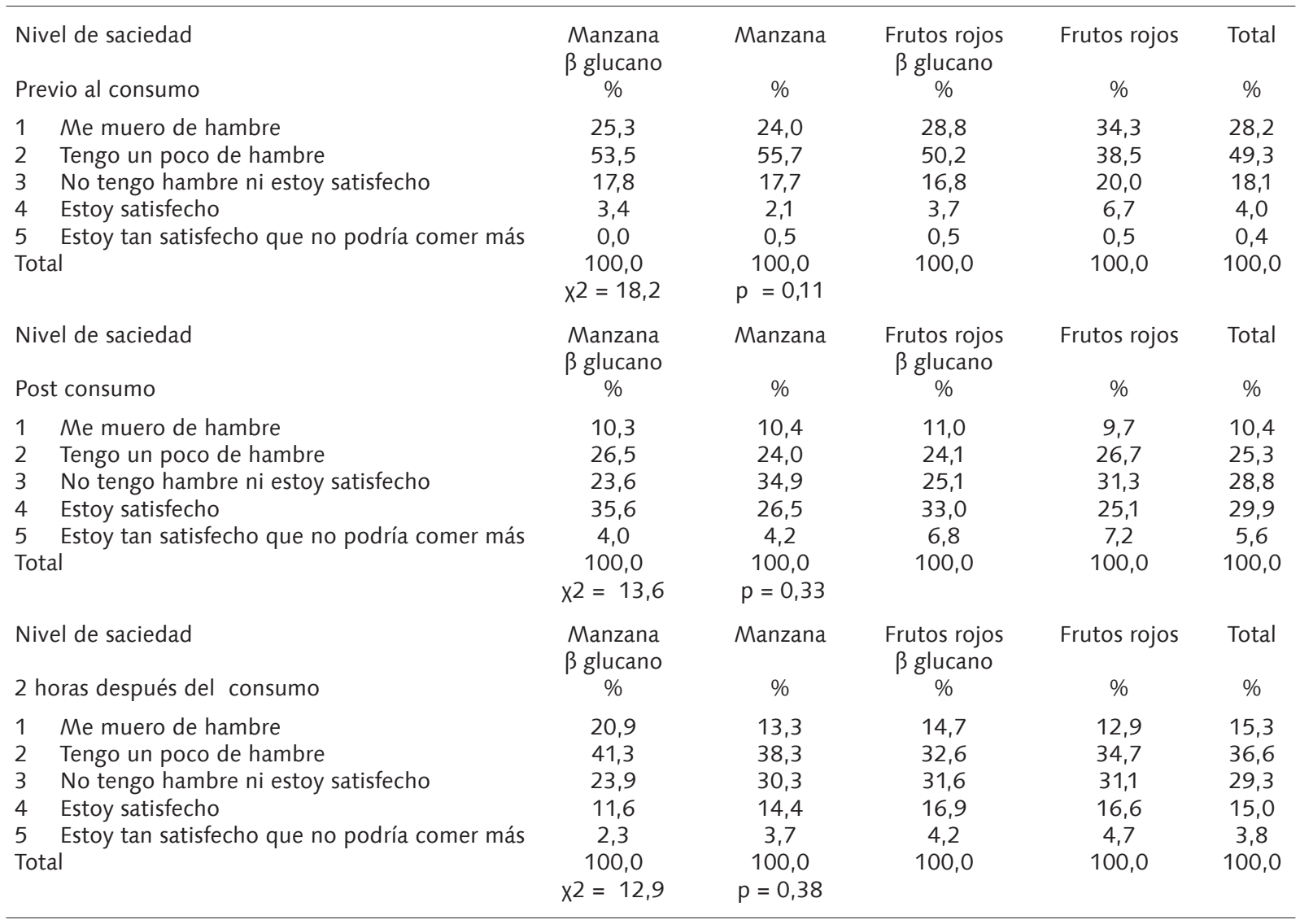

TABLA 6

Cambios en el nivel de saciedad en el tiempo.

\begin{tabular}{|c|c|c|c|c|c|c|c|}
\hline \multirow[t]{2}{*}{ Etapas } & \multicolumn{3}{|c|}{ Barritas control } & \multicolumn{3}{|c|}{ Barritas con $\beta$-glucano } & \multirow{3}{*}{$\begin{array}{l}\text { Kruskal- Wallis } \\
\text { p } \\
0,27\end{array}$} \\
\hline & p50 & & nfianza de $95 \%$ & p50 & & nfianza de 95\% & \\
\hline$t_{0}-t_{1}$ & 1 & 1 & 1 & 1 & 1 & 1 & \\
\hline t1-t2 & 0 & -1 & 0 & 0 & 0 & 0 & $<0,05$ \\
\hline$t_{0}-t_{2}$ & 0 & 0 & 0 & 0 & 0 & 0,1 & 0,26 \\
\hline
\end{tabular}


miga, ya que se oscurece, además su estructura fue más gruesa y la firmeza menor, según el estudio de Skendi y colaboradores (19). Los autores lo atribuyen a la compleja estructura molecular y forma física de las fracciones de la harina incluidas en la formulación de la masa.

Se observó que el consumo de las barritas produjo saciedad en los estudiantes, aunque en algunos casos no tuvo ningún impacto (respuesta 3: "no tengo hambre ni estoy satisfecho"), y en otros se mantuvo la sensación de hambre. Las barritas con $\beta$-glucano no produjeron mayor saciedad respecto a las barras control como se esperaba, ya que de acuerdo a la hipótesis de que $\beta$-glucano aumenta la viscosidad en el intestino delgado y retrasa la digestión de los alimentos, debería producir más saciedad (20). De acuerdo al estudio de Pentikäinen y colaboradores (21), 4 a 8 g de $\beta$-glucano tienen efecto en la saciedad, mientras que en el estudio de Hlebowicz et al. (20), se concluye que $4 \mathrm{~g}$ de $\beta$-glucano de avena en una comida semisólida no afecta la saciedad en sujetos sanos.

Después de 2 horas de haber consumido las barritas, aumenta la sensación de hambre, como se observa en la tabla 3. En el caso de las barritas de sabor manzana, la sensación de hambre (calificación 1 y 2 de la escala Escala Visual Análoga) es menor para las barritas con $\beta$-glucano respecto a la barrita control, con 51.6 y $62.2 \%$ respectivamente de respuestas para calificación 1 y 2 que indica "muero de hambre" y "un poco de hambre". En el caso de las barritas de sabor frutos rojos las respuestas fueron muy similares.

Al analizar los resultados que comparan los cambios promedios en el nivel de saciedad, se observa que en el caso de las barritas de manzana, las que contienen $\beta$-glucano y por lo tanto mayor contenido de fibra dietética, provocaron mayor saciedad después de 2 horas de haber consumido la barrita (tabla 6), aunque no fue significativa esta variación. La fibra dietética, especialmente la fibra soluble y $\beta$-glucano contribuyen a mejorar la saciedad gracias a su estructura, viscosidad y solubilidad (22-24).

El limitado efecto en la saciedad podría ser explicado porque la dosis recibida de $\beta$-glucano fue insuficiente. En este estudio no se evaluaron otros potenciales efectos metabólicos positivos, para lo cual se requieren intervenciones a más largo plazo. A pesar de ello es altamente destacable la excelente aceptabilidad de las diferentes barritas, que cumplen plenamente con una colación altamente saludable.

\section{RESUMEN}

Se desarrollaron barritas de cereal destinadas a la colación infantil en dos sabores: manzana y frutos rojos. Las barritas se elaboraron con avena y frutos deshidratados, con bajo contenido de grasas saturadas, azúcares totales y sodio. Se evaluó el efecto de la incorporación de $2.5 \%$ de $\beta$-glucano en las barritas sobre la aceptabilidad sensorial, saciedad y tolerancia digestiva en escolares de educación básica. Los resultados obtenidos demostraron alta aceptabilidad sensorial para todas las barritas (ambos sabores, con y sin $\beta$-glucano), siendo mayor en niños de $1^{\circ}$ a $4^{\circ}$ básico y en hombres $(p<0.05)$. El consumo de las barritas produjo saciedad en los niños, sin encontrase diferencias significativas por efecto de la presencia de $\beta$-glucano. Respecto a la tolerancia digestiva, no se presentaron casos de malestar, lo que indica que todas las barritas de cereal fueron bien toleradas. La incorporación de $\beta$-glucano en las barritas no afecta la aceptabilidad sensorial de éstas y tampoco afecta la saciedad respecto a barritas sin $\beta$-glucano.

Palabras clave: $\beta$-glucano, sensorial, aceptabilidad, saciedad, barrita de cereal.
Agradecimientos: Este trabajo forma parte del proyecto InnovaChile-CORFO, código 10DCO-8632 "Desarrollo de consorcio entre entidades tecnológicas de vanguardia para el desarrollo de nuevos productos funcionales en base a cereal con aporte significativo en la salud humana". Los autores agradecen la colaboración de la empresa Ideal S.A. en el desarrollo y elaboración de las barras de cereal.

\section{BIBLIOGRAFÍA}

1. Mohamed S. Functional foods against metabolic syndrome (obesity, diabetes, hypertension and dyslipidemia) and cardiovasulardisease. Trends Food Sci Technol. 2014; 35: 114-28.

2. Matsuda $M$, Shimomura I. Increased oxidative stress in obesity: Implications for metabolic syndrome, diabetes, hypertension, dyslipidemia, atherosclerosis, and cancer. Obes Res Clin Pract. 2013; 7: e330-e41.

3. Nejat EJ, Polotsky A, Pal L. Predictors of chronic disease at midlife and beyond - the health risks of obesity. Maturitas 2010; 65: 106-11.

4. Lagstrom H, Hakanen M, Niinikoski H, Viikari J, Ronnemaa $T$, Saarinen $M$ et al. Growth Patterns and Obesity Development in Overweight or Normal-Weight 13-Year-Old Adolescents: The STRIP Study. Pediatrics 2008; 122: e876-e83.

5. Ratner $R$, Hernández $P$, Martel J, Atalah E. Calidad de la alimentación y estado nutricional en estudiantes universitarios de 11 regiones de Chil. Rev Med Chil 2012; 140: 1571-9.

6. Nava MC, Pérez A, Herrera H, Hernández R. Hábitos alimentarios, actividad física y su relación con el estado nutricional-antropométrico de preescolares. Rev Chil Nutr. 2011; 38: 301-312

7. Crovetto M, Zamorano N, Medinelli A. Estado nutricional, conocimientos y conductas en escolares de kinder y primer año básico en 3 escuelas focalizadas por obesidad infantil en la comuna de Valparaíso, Chile. Rev Chil Nutr. 2010; 37: 309-20.

8. Fung C, Mclsaac J-L, Kuhle S, Kirk S, Veugelers P. The impact of a population-level school food and nutrition policy on dietary intake and body weights of Canadian children. Prevent Med. 2013; 57: 934-40.

9. Kaczmarczyk M, Miller M, Freund G. et al., The health benefits of dietary fiber: Beyond the usual suspects of type 2 diabetes mellitus, cardiovascular disease and colon cancer. Metabolism 2012; 61: 1058-66.

10. Kendall CWC, Esfahani A, Jenkins. The link between dietary fibre and human health. Food Hydrocolloids 2010; 24: 42-8

11. Othman $R$, Moghadasian $M$, Jones $P$. Cholesterol-lowering effects of oat $\beta$-glucan. Nutri Rev 2011; 69:299-309.

12. Clegg M, Thondre P. Molecular weight of barley $\beta$-glucan does not influence satiety or energy intake in healthy male subjects. Appetite 2014; 83: 167-172.

13. Kuznicki JT, Kuznicki RA, Johnson AF, Rutkiewic. Selected Sensory Methods: Problems and Approaches to Measuring Hedonics. ASTM International 1982.

14. Donadini et al., 2012 Donadini G, Fumi MD, Vanoni L, Porretta S. Hedonic response to cheese in preschoolers. J Sensory Studies 2012, 27: 176-87.

15. Blundell J, de Graaf $C$, Hulshof $T$, Jebb S, Livingstone $B$, Lluch A, Mela D, Salah S, Schuring $E$, van der Knaap $H$, Westerterp M. Appetite control: methodological aspects of the evaluation of foods. Obes Rev. 2010; 11:251-70.

16. Volikakis $P$, Biliaderis $C$, Vamvakas $C$, Zerfiridis $G$. Effects of a commercial oat- $\beta$-glucan concentrate on the chemical, 
physico-chemical and sensory attributes of a low-fat whitebrined cheese product. Food Res Internat 2004; 37: 83-94.

17. Piñero MP, Parra $K$, Huerta-Leidenz $N$, Arenas de Moreno $L$, Ferrer M, Araujo S, Barboza Y. Effect of oat's soluble fibre ( $\beta$-glucan) as a fat replacer on physical, chemical, microbiological and sensory properties of low-fat beef patties. Meat Sci 2008; 80: 675-80.

18. Amini Sarteshnizi $R$, Hosseini $H$, Bondarianzadeh $D$, Jiménez-Colmenero $F$, Khaksar R. Optimization of prebiotic sausage formulation: Effect of using $\beta$-glucan and resistant starch by D-optimal mixture design approach. LWT - Food Sci Technol. 2014; 61:1-7.

19. Skendi A, Biliaderis CG, Papageorgiou M, Izydorczyk MS. Effects of two barley $\beta$-glucan isolates on wheat flour dough and bread properties. Food Chem. 2010; 119: 159-67.

20. Hlebowicz J, Darwiche G, Björgell O, Almér L-O. Effect of Muesli with $4 \mathrm{~g}$ Oat__Glucan on Postprandial Blood
Glucose, Gastric Emptying and Satiety in Healthy Subjects: A Randomized Crossover Trial. Ame Coll Nutr. 2008; 27: 470-5.

21. Pentikäinen S, Karhunen L, Flander L, Katina K, Meynier A, Aymard P, Vinoy S, Poutanen K. Enrichment of biscuits and juice with oat $b$-glucan enhances postprandial satiety. Appetite 2014; 75: 150-6.

22. Hetherington MM, Cunningham $K$, Dye L, Gibson EL, Gregersen NT, Halford J, Van Trijp H. Potential benefits of satiety to the consumer: Scientific considerations. Nutr Res Rev. 2013; 26: 22-38.

23. Wanders A.J, van den Borne J, de Graaf $C$, Hulshof $T$, Jonathan MC, Kristensen M, Mars M, Schols HA, Feskens $E$. Effects of dietary fibre on subjective appetite, energy intake and body weight: A systematic review of randomized controlled trials. Obesity Rev. 2011; 12: 724-39.

24. Slavin J, Green H. Dietary fibre and satiety. Nutr Bull. 2007; 32: 32-42. 